\title{
VALORACIÓN Y USO DE LA MERCADOTECNIA EN LA RED HOSPITALARIA DE LA SECRETARÍA DE SALUD EN TABASCO.
}

\author{
Heberto Romeo Priego Álvarez*, Rodrigo Pavón Del Rivero**, Juan Antonio Cordova Hernández ${ }^{* * *}$.
}

\begin{abstract}
Priego-Álvarez H.R., Pavón-Del Rivero R., CórdovaHernández J.A. Valoración y uso de la mercadotecnia en la Red Hospitalaria de la Secretaría de Salud en Tabasco. Hitos de Ciencias Económico Administrativas 2012;18 (52):137-144.
\end{abstract}

\section{RESUMEN}

Objetivo: Determinar la aplicabilidad de la Mercadotecnia en los Directivos de la Red Hospitalaria de la SS Tabasco, 2011.

Material y Método: Estudio descriptivo, transversal y comparativo. Población: 74 directivos, 23 Unidades Hospitalarias. Se aplicaron dos cuestionariosCDAMH-2012 y EMIS- 2012 con escala tipo Likert (Alfa de Cronbach 0.94. El análisis de datos se realizó por medio-de estadística descriptiva, análisis factorial con índices ponderados de Aplicabilidad de la Mercadotecnia, y por otra parte se expresaron en tablas de frecuencia y gráficos. Se usó SPSS versión 15.0

Resultados: El perfil del directivo es mayormente hombres entre 36 y 55 años de edad, con estudios de posgrado (especialidad o maestría) y con antigüedad en el cargo menor a 6 años. La aplicabilidad de la Mercadotecnia, registró un índice ponderado de nivel alto en los Hospitales de Alta Especialidad y en los Comunitarios, a diferencia de los Generales donde el nivel obtenido de aplicabilidad (IPIAM) se determinó como medio. Utilizando la metodología del (EMIS 2012) se obtuvo que el puntaje promedio general de la red hospitalaria de la S. S. Tabasco fue de 80.25 , en los Hospitales de Alta Especialidad 85.38, en los Generales 78.14 y en los Comunitarios 74.16.

\author{
Priego-Álvarez H.R., Pavón-Del Rivero R., Córdova- \\ Hernández J.A. Marketing rating and use in a hospital \\ network of the secretariat of health in tabasco. \\ Hitos de Ciencias Económico Administrativas \\ 2012;18 (52):137-144.
}

\section{ABSTRACT}

Objective: Determine the applicability of the Marketing in the board of directors of the Health Secretariat hospital network of tabasco, 2011.

Material and Method: Descriptive, transversal, and comparative study. Population: 74 directors, 23 Hospital Units. Two questionnaires were applied: CDAMH-2012, and EMIS-2012 with Likert scale (Cronbach's alpha 0.94). Data analysis was performed by using descriptive statistics, factorial analysis with weighted indices of Applicability of Marketing, and on the other hand, they were expressed in frequency tables and graphs. SPSS version 15.0 was used.

Results: The profile of the manager is mostly men between 36 and 55 years old, with postgraduate studies (major or masters), and with seniority in the position of less than six years. The Applicability of Marketing registered a weighted index of high level in the high specialty hospitals and in the community ones, unlike the general hospitals where the resulting figure applicability (IPIAM) was determined as a medium. Using the methodology of (EMIS 2012) it was found that the overall average score of the hospital network of the Health Secretariat in Tabasco was 80.25; in the high specialty hospitals it was 85.38 ; in the General Hospitals, 78.14; and in the community hospitals, 74.16.

*Doctor en Ciencias de la Salud. Profesor Investigador. División Académica de Ciencias de la Salud. Universidad Juárez Autónoma de Tabasco. ** Maestro en Ciencias en Salud Pública. Directivo Médico Jurisdicción Sanitaria de Emiliano Zapata, Secretaría de Salud.

*** Maestro en Sistemas Informáticos. Profesor Investigador División Académica de Ciencias de la Salud. Universidad Juárez Autónoma de Tabasco.

Fecha de recibido: 10 de julio de 2012 Fecha de aceptación: 20 de agosto de 2012. 
Conclusiones: Los resultados obtenidos permitieron conocer que el nivel de la aplicabilidad de las estrategias mercadológicas en los hospitales de Tabasco es alta, probablemente debido a la formación académica en administración sanitaria de sus directivos.
Conclusions: The given results show that the level of the applicability of marketing strategies in the hospitals of Tabasco is high, probably due to the academic training in health administration of their managers.
Palabras clave: Mercadotecnia Sanitaria. Directivos de hospitales.
Key words: Healthcare marketing. Hospital board of Directors.

\section{DIRECCIÓN PARA RECIBIR CORRESPONDENCIA: Correo electrónico: heberto_priego@hotmail.com}

a Mercadotecnia Hospitalaria, es la gestión de un intercambio entre el hospital y los ciudadanos (usuarios potenciales y reales) para conseguir el bienestar deseado, ésta orientación tiene como clave la determinación de las necesidades y valores de la población objetivo (mercado meta), a fin de conseguir el deseado nivel de satisfacción. Pero esta adaptación, «no se estructura en base a logotipos y slogans, no basta con crear servicios de información al usuario. No es suficiente con llenarnos la boca con la palabra cliente. Es necesario dar un vuelco, un cambio, una reorientación» (March, Priego, Prieto, 1996, pp. 9-12).

En la actualidad, los hospitales tanto públicos como privados, de los países desarrollados cuentan con una unidad de mercadotecnia que aglutina los tradicionales departamentos de relaciones públicas, planeación, trabajo social, admisión hospitalaria y de calidad. Son funciones primordiales de dicha unidad el desarrollo de acciones mercadológicas, tanto externas como internas, que garanticen la calidad en la prestación de los servicios, que mantengan sensible a la organización hacia la satisfacción de las necesidades de los usuarios y tengan un estado que permanente alerta hacia los cambios del entorno que afecten el mercado.

Existen departamentos en los hospitales que realizan actividades de mercadotecnia, como el de relaciones públicas y planeación, situación que ha confundido a los directivos de los hospitales debido a que las actividades de publicidad y relaciones públicas las toman como equivalentes de mercadotecnia. (Naidu et al., 1992, pp. 29-43).

Sin embargo, no es conveniente asignar las actividades de mercadeo a un departamento ya establecido, sino por el contrario crear un nuevo departamento o área denominada de mercadotecnia que cuente con personal altamente calificado para estudiar el mercado, productos y territorios, considerando que la mercadotecnia hospitalaria debe: a) proporcionar medios para programar nuevos servicios, b) icrementar el uso de programas existentes, c) identificar a los usuarios reales y potenciales, d) generar información oportuna para la toma de decisiones, e) ofertar los servicios requeridos por el mercado meta, $\mathrm{f}$ ) establecer lineamientos para la prestación de los servicios, g)hacer análisis de competencia (participación del mercado y posicionamiento de hospitales, h) realizar programa de capacitación para el desarrollo de la mercadotecnia interna e interactiva, i) efectuar estudios y pronósticos de la demanda por servicios, y l) desarrollar programas de calidad. (Burton, 1991, pp. 83-84).

Para llevar a cabo tales actividades mercadotécnicas es conveniente regirse por ciertos principios éticos como los mencionados por Goldman: ver primero el bienestar del paciente, evitar servicios innecesarios, mantener altos estándares de honestidad y precisión, y ser responsables con el público (población usuaria). (Goldman, 1993).

La Mercadotecnia Social Hospitalaria, ha sido objeto de continua atención por parte de los investigadores, tal interés encuentra en gran medida justificación al considerar que la aplicabilidad de la mercadotecnia puede generar ventajas competitivas y con ello un impacto positivo en sus resultados. Cuando se habla de la utilización de una estrategia de mercadotecnia social se está pensando no en una estrategia para copiar, sino en una práctica; no en una solución mágica para los problemas, sino en la consecución de un proceso, objetivo y científicamente argumentado que permita alcanzar el éxito. (Suárez, 2007, pp. 182-187).

El presente estudio da continuidad a este tipo de investigaciones que generan un conocimiento del sector salud en el ámbito hospitalario y de las condiciones en las que se da la Mercadotecnia, ya que si bien existen estudios realizados en otros contextos (White, 2001, pp. 327-336), se requiere la determinación de la aplicabilidad de la mercadotecnia en los Directivos de la Red Hospitalaria de la Secretaría de Salud en Tabasco (SS Tabasco).

\section{MATERIAL Y MÉTODO}

El estudio realizado es descriptivo transversal comparativo. Se consideró como población en estudio a los directivos de los hospitales de la Red Hospitalaria de la SS del Estado de Tabasco. Se incluyeron los 
23 hospitales que conforman la red de servicios de atención de laSS Tabasco. La muestra consideró a 3 y/o 4 de los directivos $(n=73)$ de cada uno de los hospitales incluyendo los tres tipos de atención clasificados: Comunitarios, Generales y de Alta Especialidad, que ejercieran funciones directivas a nivel de mando medio y/o superior (Director, Subdirector y/o Coordinador Médico y/o Coordinador de Calidad y Educación en Salud y SubdirectorAdministrativo). Se excluyeron del estudio a los directivos que no desearon participar en el proyecto de investigación y aquellos que no estaban en funciones y/o ocupando su cargo temporalmente en el momento de la entrevista (Licencia, permisos o incapacidad).

Se utilizaron 2 instrumentos de recolección de información. El primero, denominado «Caracterización de los Directivos y Aplicabilidad de la Mercadotecnia Hospitalaria» (CDAMH-2012). Instrumento diseñado por PriegoÁlvarez et als, para la determinación del uso de la mercadotecnia en profesionales de la salud (enfermeras y nutriólogos) (Priego, Córdova, Lara, 2011, pp. 46-57); el cual fue modificado con la inclusión de más preguntas relacionadas a determinar el perfil académico e intereses del directivo. El segundo, denominado «Enfoque de la mercadotecnia en las instituciones de salud» (EMIS-2012) propuesto por Nery Suárez Lugo en 2004 en Cuba (Suárez, 2004), estructurado en 20 ítems distribuido en 10 categorías para valorar la aplicabilidad general de la mercadotecnia en una unidad hospitalaria. La validación del instrumento auto-administrado se realizó midiendo la confiabilidad de la consistencia interna a través de la prueba del Alpha de Cronbach (resultado de 0.94). La idea de poder contar con dos instrumentos de recolección sobre una misma población tuvo como objeto confirmar la aplicabilidad de la mercadotecnia, tanto a nivel individual (directivo) como en el ámbito organizacional (hospital).

Con base a la información recolectada en el cuestionario CDAMH-2012 se construyó el Índice Ponderado Integrado de Aplicabilidad de la Mercadotecnia en unidades hospitalarias (IPIAM), el cual determina el nivel de aplicación de la mercadotecnia por tipo de unidad hospitalaria (Hospitales deAlta Especialidad, Hospitales Generales y Hospitales Comunitarios), valorándose en las categorías Bajo, Medio y Alta.

\section{RESULTADOS}

\section{Caracterización de los directivos}

La muestra comprendió 74 directivos, de los cuales 32 (43\%) laboraban en hospitales Generales, 24 (32\%) eran de los hospitales Comunitarios, y los restantes $18(24 \%)$ ocupaban cargos en los hospitales de alta especialidad.

Las características sociodemográficas de la muestra seleccionada se describen en la Tabla I. El perfil de los directivos encuestados corresponde mayoritariamentea hombres (62\%), entre los 36 y 55 años de edad (66\%), con antigüedad menor a 6 años en el cargo directivo $(82 \%)$, y con estudios de posgrado (62\%). De los que

\section{TABLA I CARACTERÍSTICAS SOCIODEMOGRÁFICAS DE LOS DIRECTIVOS DE LA RED HOSPITALARIA DE LA SECRETARÍA DE SALUD EN TABASCO.}

\begin{tabular}{|c|c|c|}
\hline Características & $n=74$ & Porcentaje (\%) \\
\hline \multicolumn{3}{|l|}{ Sexo } \\
\hline Masculino & 46 & 62 \\
\hline Femenino & 28 & 38 \\
\hline \multicolumn{3}{|l|}{ Edad del entrevistado (años) } \\
\hline $25-35$ años & 18 & 24 \\
\hline $36-45$ años & 22 & 30 \\
\hline $46-55$ años & 26 & 35 \\
\hline$>\quad 55$ años & 8 & 11 \\
\hline \multicolumn{3}{|l|}{ Perfil Académico Profesional } \\
\hline Licenciatura & 28 & 38 \\
\hline Especialidad & 14 & 19 \\
\hline Maestría & 32 & 43 \\
\hline \multicolumn{3}{|l|}{ Cargo Laboral. } \\
\hline Director & 23 & 31 \\
\hline Subdirector o Coordinador & 28 & 38 \\
\hline Subdirector Administrativo & 23 & 31 \\
\hline \multicolumn{3}{|l|}{ Tiempo de Ejercer el Cargo } \\
\hline$<3$ años & 41 & 55 \\
\hline $4-6$ años & 20 & 27 \\
\hline$>6$ años & 13 & 18 \\
\hline
\end{tabular}

Fuente: Encuesta a directivos sobre aplicabilidad de la Mercadotecnia Hospitalaria (CDAMH-2012). 
contaban con Maestría, 17 eran egresados de la Maestría de Administración de Hospitales, 10 de la Maestría en Salud Pública con énfasis en administración y 5 de la Maestría en Servicios o Sistemas de Salud. Es importante mencionar que de aquellos que sólo contaban con estudios de licenciatura provenían de las Ciencias Económico Administrativas y eran mayormente subdirectores administrativos. Solo dos directores médicos no tenían estudios de posgrado.

La mayoría de los directivos encuestados (50\%) consideraron que su principal función dentro del proceso administrativo de los hospitales se relacionaba propiamente con su actividad directiva (ejecución). Los aspectos de organización (coordinación) fueron los segundos en orden de importancia en ser considerados (30\%). El control de los procesos (verificación) se estimó en tercer lugar en importancia (15\%). Los menormente considerados (5\%) fueron los aspectos de planeación (objetivos y metas).

\section{Aplicabilidad de la mercadotecnia}

La Aplicabilidad de la Mercadotecnia en la Red Hospitalaria de la Secretaría de Salud en Tabasco se determinó con base al Índice Ponderado General de la Aplicación de la Mercadotecnia hospitalaria (IPGAM) obteniéndose un nivel de aplicabilidad alto (ver Tabla II).

Con base a la metodología diseñada por Suárez Lugo y recolectada en el formato EMIS-2012, se estableció que la aplicabilidad de la Mercadotecnia registrada más alta (85.38 puntos) fue en los 5 Hospitales de Alta Especialidad, donde los informantes fueron 18 directivos. En cuanto a los 10 Hospitales Generales, el resultado fue de 78.74 puntos, y se aplicó el cuestionario a 32 directivos; finalmente el resultado de los 8 hospitales comunitarios fue de 74.17 puntos con 24 directivos encuestados (ver Figura 1).

El puntaje obtenido para los hospitales de Alta Especialidad de 85.38, significa que la institución toma en consideración al usuario como su razón de ser, pero tiene muchos aspectos que debe mejorar. El hospital de Salud Mental y el Hospital Dr. Gustavo A. Rovirosa Pérez estuvieron por arriba de la media aritmética del grupo (90.25 y 87.25 respectivamente). Respecto a las Categorías relacionadas con el instrumento EMIS-2012 se observó que los ítems mejor evaluados fueron los que se refieren a la definición de la misión y la visión, la institución está orientada a la satisfacción de las necesidades y la relación de la calidad de los servicios con los indicadores de salud. Aunque también en la categoría de siempre, la mayor frecuencia corresponde a apuntar que la institución dispone de un sistema de recolección de información que permite verla desde afuera, investigando el planteamiento del cliente externo y su grado de satisfacción, de tal manera que el cliente interno en su entrenamiento y capacitación incluye el manejo de las relaciones con el usuario (ver Tabla III).

El puntaje obtenido para los hospitales Generales fue de 78.14 , lo que significa que la institución no ofrece un servicio de calidad porque no toma en consideración los trabajadores que ofrecen el servicio y los usuarios. Debe de revisar su razón de ser y centrarse en la solución de los problemas. El Hospital de Paraíso tuvo el puntaje menor (62.33), 15.81 puntos por debajo de la media aritmética. Por el contrario hubieron seis hospitales que estuvieron por arriba de la media: Hospital de Huimanguillo (92.33 punto), Hospital de E. Zapata

TABLA II. ÍNDICE PONDERADO GENERAL DE LA APLICACIÓN DE LA MERCADOTECNIA HOSPITALARIA (IPGAM)

\begin{tabular}{|c|c|c|c|c|c|c|c|c|}
\hline \multirow{2}{*}{$\begin{array}{l}\text { Aplicación del MKT } \\
\qquad \mathrm{N}=74\end{array}$} & \multicolumn{2}{|c|}{ sí } & \multicolumn{2}{|c|}{ NO } & \multicolumn{4}{|c|}{ Variables } \\
\hline & $f$ & $\%$ & $f$ & $\%$ & $\begin{array}{c}\text { Valor } \\
\text { real }\end{array}$ & $\begin{array}{c}\text { Valor } \\
\text { estimado }\end{array}$ & Categoría & $\begin{array}{c}\text { Nivel de } \\
\text { aplicabilidad }\end{array}$ \\
\hline $\begin{array}{l}\text { En la gerencia de } \\
\text { hospitales }\end{array}$ & 68 & 92 & 6 & 8 & .75 & 3 & A & \\
\hline $\begin{array}{l}\text { En el área profesional del } \\
\text { directivo }\end{array}$ & 67 & 91 & 7 & 9 & .75 & 3 & A & $=11$ \\
\hline $\begin{array}{l}\text { Utilización de estrategias } \\
\text { mercadológicas } \\
\text { hospitalarias }\end{array}$ & 66 & 89 & 8 & 11 & .75 & 3 & A & ALTO \\
\hline $\begin{array}{l}\text { En la of erta de servicios } \\
\text { por los clientes internos }\end{array}$ & 37 & 50 & 37 & 50 & .50 & 2 & M & \\
\hline
\end{tabular}

Fuente: Encuesta a directivos sobre aplicabilidad de la Mercadotecnia Hospitalaria (CDAMH-2012). 
FIGURA 1. APLICABILIDAD DE LA MERCADOTECNIA EN LA RED HOSPITALARIA S.S. TABASCO

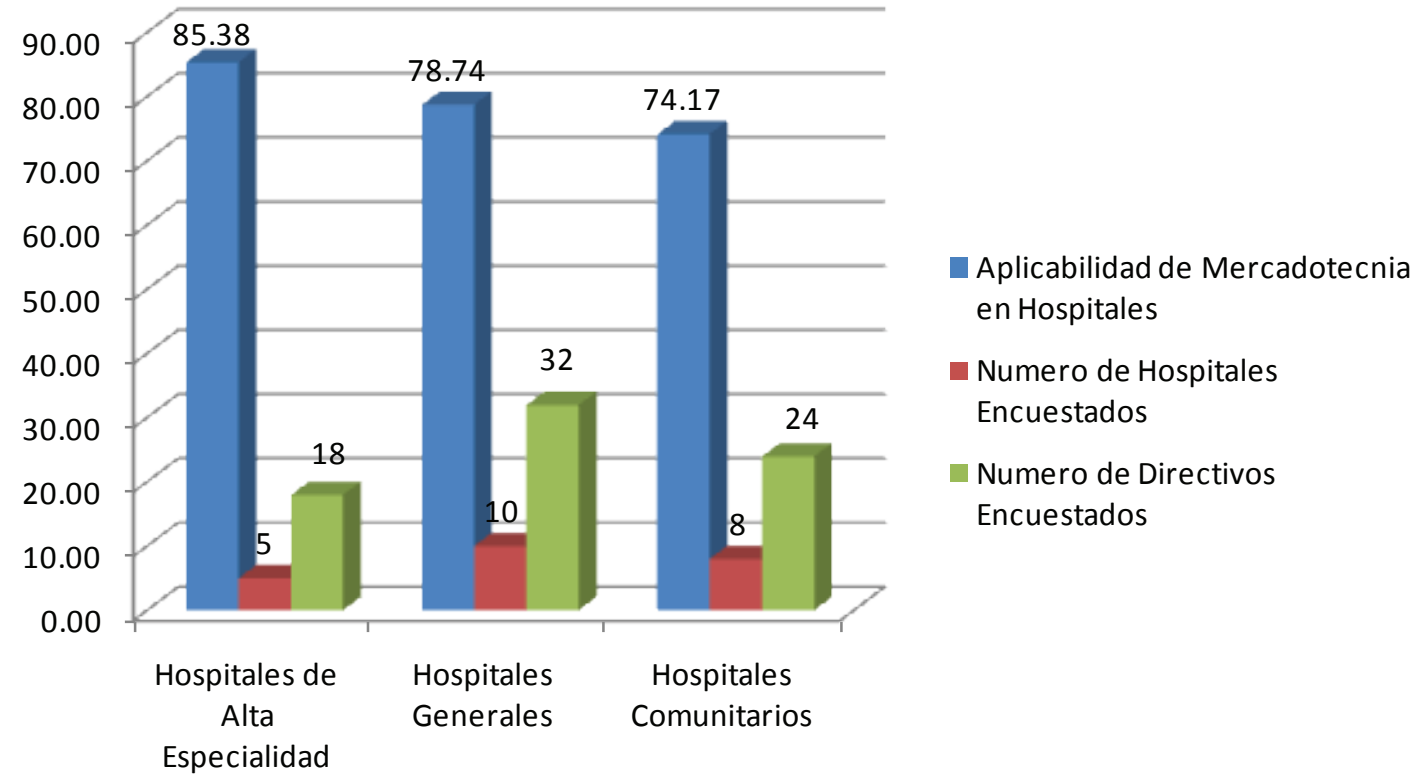

Fuente: Encuesta a directivos sobre enfoque de la Mercadotecnia en las Instituciones de Salud (EMIS-2012).

(85.50), Hospital de Comalcalco (84 puntos), Hospital Villa Benito Juárez (80.67), Hospital de Cárdenas (80.25) y el Hospital de Cunduacán (79.33 puntos).

Respecto a las categorías relacionadas con el instrumento EMIS -2012 se observó que, los ítems mejor evaluados fueron los que se refieren a que la institución está orientada a la satisfacción de las necesidades y la relación de la calidad de los servicios con los indicadores de salud y reconoce que calidad es igual a satisfacción de los usuarios y pacientes.
Y también en la categoría de siempre, la media corresponde a apuntar que la institución dispone de un sistema de recolección de información que permite verla desde afuera, investigando el planteamiento del cliente externo y su grado de satisfacción, así el cliente interno en su entrenamiento y capacitación incluye el manejo de las relaciones con el usuario (ver Tabla IV).

Con respecto a los hospitales Comunitarios el puntaje obtenido fue de 74.16 , lo que significa que la institución

TABLA III. CATEGORÍAS DE LOS SERVICIOS EN HOSPITALES ALTA ESPECIALIDAD S.S. TABASCO

\begin{tabular}{|c|c|c|c|c|c|c|c|}
\hline Categoría & Nunca & $\begin{array}{c}\text { Casi } \\
\text { nunca }\end{array}$ & $\begin{array}{l}\text { Pocas } \\
\text { veces }\end{array}$ & $\begin{array}{l}\text { Algunas } \\
\text { veces }\end{array}$ & Habitualmente & Siempre & Total \\
\hline Calidad con enfoque de usuario & -_- & -_- & -_- & -_- & $44.4 \%$ & $55.6 \%$ & $100 \%$ \\
\hline Organización con enfoque de usuario & -_- & --_ & _-_ & $11.1 \%$ & $22.2 \%$ & $66.7 \%$ & $100 \%$ \\
\hline Proceso con enfoque de usuario & -_- & -_- & -_- & $5.6 \%$ & $61.1 \%$ & $33.3 \%$ & $100 \%$ \\
\hline Resultado / satisfacción usuario & -_- & -_- & $5.6 \%$ & $11.1 \%$ & $33.3 \%$ & $50.0 \%$ & $100 \%$ \\
\hline Condiciones materiales & - & -_- & $5.6 \%$ & $5.6 \%$ & $38.9 \%$ & $50.0 \%$ & $100 \%$ \\
\hline Quejas & - & --_ & -_- & $11.1 \%$ & $22.2 \%$ & $66.7 \%$ & $100 \%$ \\
\hline Valoración mercadotecnia & -_- & --_- & $5.6 \%$ & $16.6 \%$ & $27.8 \%$ & $50.0 \%$ & $100 \%$ \\
\hline Satisfacción usuario & -_- & -_- & $5.6 \%$ & $5.6 \%$ & $27.8 \%$ & $61.0 \%$ & $100 \%$ \\
\hline Satisfacción trabajadores & -- & - & $11.1 \%$ & $5.6 \%$ & $44.4 \%$ & $38.9 \%$ & $100 \%$ \\
\hline Capacitación trabajadores & - & _-_ & $5.6 \%$ & $5.6 \%$ & $27.8 \%$ & $61.0 \%$ & $100 \%$ \\
\hline
\end{tabular}

Fuente: Encuesta a directivos sobre enfoque de la Mercadotecnia en las Instituciones de Salud (EMIS-2012). 
TABLA IV. CATEGORÍAS DE LOS SERVICIOS EN HOSPITALES GENERALES S.S. TABASCO

\begin{tabular}{|c|c|c|c|c|c|c|c|}
\hline Categoría & Nunca & $\begin{array}{c}\text { Casi } \\
\text { nunca }\end{array}$ & $\begin{array}{l}\text { Pocas } \\
\text { veces }\end{array}$ & $\begin{array}{c}\text { Algunas } \\
\text { veces }\end{array}$ & Habitualmente & Siempre & Total \\
\hline Calidad con enfoque de usuario & - & -_- & _-_ & $9.4 \%$ & $37.5 \%$ & $53.1 \%$ & $100 \%$ \\
\hline Organización con enfoque de usuario & - & -_- & $3.2 \%$ & $15.6 \%$ & $40.6 \%$ & $40.6 \%$ & $100 \%$ \\
\hline Proceso con enfoque de usuario & _- & -_- & -_- & $18.8 \%$ & $59.3 \%$ & $21.9 \%$ & $100 \%$ \\
\hline Resultado / satisfacción usuario & --- & $3.1 \%$ & -_- & $12.5 \%$ & $40.6 \%$ & $43.8 \%$ & $100 \%$ \\
\hline Condiciones materiales & - & $3.1 \%$ & $6.3 \%$ & $12.5 \%$ & $46.8 \%$ & $31.3 \%$ & $100 \%$ \\
\hline Quejas & - & --- & $6.3 \%$ & $9.4 \%$ & $34.4 \%$ & $50.0 \%$ & $100 \%$ \\
\hline Valoración mercadotecnia & -_- & -_- & $3.1 \%$ & $18.8 \%$ & $56.3 \%$ & $21.9 \%$ & $100 \%$ \\
\hline Satisfacción usuario & --- & -_- & $9.4 \%$ & $15.6 \%$ & $50.0 \%$ & $25.0 \%$ & $100 \%$ \\
\hline Satisfacción trabajadores & - & - & $12.5 \%$ & $15.6 \%$ & $59.4 \%$ & $12.5 \%$ & $100 \%$ \\
\hline Capacitación trabajadores & - & -_- & $18.8 \%$ & $15.6 \%$ & $46.8 \%$ & $18.8 \%$ & $100 \%$ \\
\hline
\end{tabular}

Fuente: Encuesta a directivos sobre enfoque de la Mercadotecnia en las Instituciones de Salud (EMIS-2012).

no ofrece un servicio de calidad porque no toma en consideración los trabajadores que ofrecen el servicio y los usuarios. Debe de revisar su razón de ser y centrarse en la solución de los problemas. El puntaje menor observado fue para el hospital de Centla con 49 puntos, con una diferencia de casi 25 puntos respecto de la media del grupo. El hospital con puntaje más alto se registró en Jalapa (93 puntos) con 18.84 puntos por arriba de la media grupal. Los otros hospitales por arriba de la media fueron el de Tenosique y el de Jalpa de Méndez con registros de 83.33 y 81.33 puntos, respectivamente.

Dentro de las categorías los ítems mejor evaluados fueron los que se refieren a que calidad es igual a satisfacción de los usuarios y pacientes, a reconocer la definición de la misión y la visión, también a que la institución está orientada a la satisfacción de las necesidades y la relación de la calidad de los servicios con los indicadores de salud (ver Tabla V).

\section{Usos y aplicaciones específicas de la mercadotecnia hospitalaria.}

A la comparación de medias aritméticas se observó que el mayor uso de la mercadotecnia se registró en los Hospitales Generales (HG). Con relación a al ámbito de aplicación de la mercadotecnia, el mayor uso del marketing externo se identificó en los Hospitales Comunitarios $(\mathrm{HC})$, y el marketing interno en los Hospitales de Alta Especialidad (HAE). El uso de la MKT en la Red Hospitalaria de la SS Tabasco registra una media de 72.09. La aplicación de la mercadotecnia externa registrada se asoció mayormente a mejoras en la imagen de salud y la atracción de recursos y personas. El marketing interno se relacionó más con el mejor empleo de los recursos existentes (ver Tabla VI).

\section{DISCUSIÓN}

En los resultados obtenidos de la presente investigación, se encontró que en lo relacionado al perfil de los directivos de la red hospitalaria de la SS-Tabasco es la de ser mayormente masculino, entre 36 y 55 años de edad, con estudios de posgrado (prioritariamente Maestría en Administración de Hospitales). Al compararlo con un estudio similar realizado en Cuba, se encuentran coincidencias en edad y estudios de posgrado, aunque contrasta en el sexo predominante (en Cuba los directivos eran mayormente del sexo femenino) (Suárez, 2008).

La aplicabilidad de la Mercadotecnia, registró un índice ponderado de nivel alto en los Hospitales de Alta Especialidad y en los Hospitales Comunitarios, a diferencia de los Hospitales Generales donde el nivel obtenido de aplicabilidad (IPIAM), se determinó como medio. Este alto nivel de aplicabilidad de la Mercadotecnia Hospitalaria identificado, pudiera ser explicable a la formación administrativa de sus directivos, ya que como es sabido el conocimiento adquirido en programas de formación académica facilita la implementación y desarrollo de estrategias mercadológicas en el Sector Salud de Tabasco (García 
TABLA V. CATEGORÍAS DE LOS SERVICIOS EN HOSPITALES COMUNITARIOS S.S. TABASCO

\begin{tabular}{|c|c|c|c|c|c|c|c|}
\hline Categoría & Nunca & $\begin{array}{c}\text { Casi } \\
\text { nunca }\end{array}$ & $\begin{array}{l}\text { Pocas } \\
\text { veces }\end{array}$ & $\begin{array}{c}\text { Algunas } \\
\text { veces }\end{array}$ & Habitualmente & Siempre & Total \\
\hline Calidad con enfoque de usuario & --- & - & -_- & $25.0 \%$ & $20.8 \%$ & $54.2 \%$ & $100 \%$ \\
\hline Organización con enfoque de usuario & -- & -- & $8.4 \%$ & $20.8 \%$ & $20.8 \%$ & $50.0 \%$ & $100 \%$ \\
\hline Proceso con enfoque de usuario & - & -_- & $12.5 \%$ & $25.0 \%$ & $41.7 \%$ & $20.8 \%$ & $100 \%$ \\
\hline Resultado / satisfacción usuario & - & 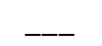 & --- & $33.4 \%$ & $20.8 \%$ & $45.8 \%$ & $100 \%$ \\
\hline Condiciones materiales & - & $4.2 \%$ & $12.5 \%$ & $20.8 \%$ & $20.8 \%$ & $41.7 \%$ & $100 \%$ \\
\hline Quejas & - & -_- & -_- & $45.8 \%$ & $8.4 \%$ & $45.8 \%$ & $100 \%$ \\
\hline Valoración mercadotecnia & $12.5 \%$ & $8.3 \%$ & $12.5 \%$ & $20.8 \%$ & $16.7 \%$ & $29.2 \%$ & $100 \%$ \\
\hline Satisfacción usuario & - & -_- & $12.5 \%$ & $33.3 \%$ & $29.2 \%$ & $25.0 \%$ & $100 \%$ \\
\hline Satisfacción trabajadores & - & -_- & $8.4 \%$ & $37.5 \%$ & $37.5 \%$ & $16.6 \%$ & $100 \%$ \\
\hline Capacitación trabajadores & $4.2 \%$ & $8.3 \%$ & $12.5 \%$ & $16.7 \%$ & $20.8 \%$ & $37.5 \%$ & $100 \%$ \\
\hline
\end{tabular}

Fuente: Encuesta a directivos sobre enfoque de la Mercadotecnia en las Instituciones de Salud (EMIS-2012).

TABLA VI. USOS Y APLICACIONES ESPECÍFICAS DE LA MERCADOTECNIA HOSPITALARIA S.S. TABASCO

\begin{tabular}{lccccc}
\hline \multicolumn{1}{c}{ Tipo de Hospital } & HAE & HG & HC & Hosp. \\
\hline Características & $\dot{\mathbf{X}}$ & $\dot{\mathbf{X}}$ & $\dot{\mathbf{X}}$ & \\
\cline { 1 - 4 } Uso de la MKT en la red hospitalaria & 76.39 & $\mathbf{7 8 . 1 3}$ & 61.76 & $\mathbf{7 2 . 0 9}$ \\
Aplicación de MKT en la red hospitalaria & & & & \\
Externas & 68.63 & 66.67 & $\mathbf{7 0 . 3 7}$ & 68.55 \\
$\begin{array}{l}\text { Interna } \\
\text { Fuente: Encuesta a directivos sobre aplicabilidad de la Mercadotecnia Hospitalaria (CDAMH-2012). }\end{array}$
\end{tabular}

\& Priego, 2004, pp. 21-26), debido a que los administradores de los servicios de salud presentan actitudes positivas hacia la mercadotecnia (Burton, 1991, pp. 81-95). Este aspecto formativo de los directivos en la aplicación de la mercadotecnia social es también reconocido como una herramienta para incrementar la eficacia en los planes de salud pública (Boerti, 2008, pp. 27-36). Cabe hacer notar que el contar con estudios de posgrado en el campo de la administración hospitalaria o equivalente, es una condición a cubrir para ocupar el cargo de director de unidad hospitalaria dentro de la Secretaría de Salud en Tabasco, por lo que para el cumplimiento de las funciones del Director Hospitalario debería llenarse el requisito establecido.
A la comparación de los resultados obtenidos en la presente investigación con estudios de metodología similar, cabe señalar que el puntaje promedio general de la Red Hospitalaria de la Secretaría de Salud de Tabasco fue de 80.25, cifra muy superior a la registrada a la aplicabilidad de la Mercadotecnia de 64.50 en los hospitales del sistema de salud cubano (Suárez, 2008).

Los puntajes más altos de aplicación de la mercadotecnia en la red hospitalaria de la SS-Tabasco (entre 90 y 99 puntos) se registraron solo en el $13 \%$ de los hospitales, similar al $17 \%$ obtenido en el Sistema de Salud Cubano. Sin embargo, existen diferencias notorias en cuanto al enfoque de calidad que es 
mayormente reconocido en la red hospitalaria de la SS-Tabasco. Al respecto, cabe apuntar que el $43.50 \%$ de las Instituciones de la Red Hospitalaria del Estado de Tabasco, obtuvieron una puntuación entre 80 y 89 reflejándose que los usuarios son la razón de ser de las mismas pero que tienen muchos aspectos en los que deben mejorar, a diferencia del estudio realizado en Cuba que obtuvo un resultado menor de $25.80 \%$. Al respecto debe señalarse que la comparación de los resultados obtenidos en el instrumento en su forma de auto-diagnóstico, permite considerar la posibilidad de obtener resultados sobreestimados en su valoración, dado que se está diagnosticando una actividad en la cual los informantes se encuentran involucrados.

Los hospitales con nivel de aplicación alto de la Mercadotecnia, no se concentran en un grupo en específico (se esperaba que fueran los hospitales de alta especialidad los que tuvieran un mayor uso de estrategias mercadológicas dada su alta complejidad y mayor dotación de recursos), sino que existen punteros por cada grupo de hospitales: el Hospital de Salud Mental en el caso de los de Alta Especialidad, el Hospital de Huimanguillo en el grupo de los Hospitales. Generales, y el Hospital de Jalapa en los Hospitales Comunitarios.

Las categorías de mayor peso obtenidas para la aplicación de la Mercadotecnia en la Red Hospitalaria de la Secretaría de Salud en Tabasco, fueron la calidad y la organización con enfoque de usuario, lo que resulta coincidente con una investigación similar realizada en un hospital del Sur de Chile en la que se asoció la orientación de objetivos, planes y programas de funcionamiento del hospital para la satisfacción de sus usuarios con los componentes de cultura orientada hacia la valoración del usuario y la gestión de recursos humanos y atención de usuarios. (Rivero, 2006, pp. 353-360).

\section{CONCLUSIONES}

Los resultados obtenidos permitieron conocer que el nivel de la aplicabilidad de las estrategias mercadológicas en la Red Hospitalaria de la Secretaría de Salud es alto, probablemente debido a la formación académica en administración sanitaria de sus directivos. La normatividad institucional establece un perfil directivo formado en administración lo que necesariamente coadyuva al uso del marketing tanto externo como interno. La calidad y la organización con enfoque de usuario son las categorías identificadas como de mayor peso en la aplicabilidad de la mercadotecnia hospitalaria en la SS-Tabasco.

\section{REFERENCIAS}

March, J.C., Priego, H, y Prieto, M.A. (1996). Consideraciones mercadológicas en la gestión hospitalaria. Revista Hitos de la Ciencia Económico-Administrativa, 3, 9-12.

Naidu, G. et al. (1992). Organization of marketing in US hospitals: an empirical investigation. Health Care Management Review, 17(4), 29-43.

Burton, G.E. (1991). Health Care Advertising. Comparing the attitudes of hospital administrators and consumers. Journal of Hospital Marketing, 5(2), 83-84.

Goldman, A. (1993). Philosophical applications of cognitive science. San Francisco, US.

Suárez, N. (2007). Enfoque social de la mercadotecnia sanitaria. (pp. 182-187). La Habana: Editorial Ciencias Médicas.

White R., Thompson M., \& Patel B. (2001). Hospital Marketing Orientation and Managed Care Processes: Are they coordinated? Journal of Health Care Management ,46(5), 327-336.

Fonseca N., y Mejía R. (2002). Análisis de los usos de la mercadotecnia hospitalaria en México (Tesis Doctoral). Universidad Nacional Autónoma de México, México.

Gbadeyan R.A. (2010). Health care marketing and public relations in not for profit hospitales in Nigeria. International Journal of Business and Management, 5(7), 117-125.

Sharma, A., Dadwal, S. S., \& Mahal S. S. (2008). Why hospitals also need benchmarking marketing capabilities? An empirical study of relationships between marketing practices and its outcomes. Paradigm, 12(1), 77-84.

Priego, H.R., Córdova, J.A, y Lara, M.A. (2011). La mercadotecnia en el ejercicio profesional de la enfermería en Tabasco (México). Pensamiento \& Gestión, 30 (1), 46-57.

Suárez, L. N. (2004). Estudio sobre conocimientos, actitudes y prácticas de mercadotecnia en el sistema nacional de salud de Cuba. Informe de Investigación. Escuela Nacional de Salud Pública. MINSAP.

Suárez, L. N. (2008). Mercadotecnia en el Sistema Nacional de Salud de Cuba. Revista Cubana de Salud Pública, 34(1).

García, R., y Priego, H. R. (2004). El Rol en el sistema de salud y las actitudes sobre la mercadotecnia. El caso de directivos del sector salud. Hitos de Ciencias Económico Administrativas, 26, 21-26.

Burton, G.E. (1991). Medical doctors and consumers view medical advertising. Health Marketing Quarterly, 9(1-2), 81-95.

Mc Dermott, D.R. \& Little, M.W. (1988). Hospital administrator's perspectives regarding the health care services. Health Marketing Quarterly, 5(3-4), 61-73.

Boerti, P. A., Martín, S. J.D. \& Porta, M. (2008). El marketing como herramienta para incrementar la eficacia de los planes de salud pública. Gaceta Sanitaria, 22 (Supl. 1), 27-36.

Riveros, S, J., y Berné, M. C. (2006). La aplicación del marketing en hospitales públicos desde la perspectiva de los funcionarios: El caso de un hospital del sur de Chile. Revista Médica de Chile, 3(134), 353-360.

HITOS DE CIENCIAS ECONÓMICO ADMINISTRATIVAS 